\title{
Therapeutic Hypothermia: Its Potential and Questionable Role in Traumatic Brain Injury
}

Tieng Kong $\mathrm{RL}^{1^{*}}$

${ }^{1}$ University of British Columbia, 2329 West Mall, Vancouver BC V6T 1Z4, Canada

Corresponding Author: Rowena Li Tieng Kong

Address: University of British Columbia, 2329 West Mall, Vancouver BC V6T 1Z4, Canada; E-mail: rowena.kong@alumni.ubc.ca

Received date: 14 October 2019; Accepted date: 24 October 2019; Published date: 30 October 2019

Citation: Tieng Kong RL. Therapeutic Hypothermia: Its Potential and Questionable Role in Traumatic Brain Injury. Asp Biomed Clin Case Rep. 2019 Oct 30;2(2):84-86.

Copyright (C) 2019 Tieng Kong RL. This is an open-access article distributed under the Creative Commons Attribution License, which permits unrestricted use, distribution, and reproduction in any medium provided the original work is properly cited.

\section{Keywords}

Therapeutic Hypothermia; Temperature; Cooling; Intracranial Hypertension; Traumatic Brain Injury

From the acute management of cardiac arrest and stroke to traumatic brain injury, the implementation of hypothermia therapy since the past two decades has progressed from various guidelines committees' consideration of minimal benefit to mixed favorable outcomes being obtained from numerous randomized controlled trials [1]. As far as cases of traumatic brain injury are concerned, there were evidences of positive benefit in terms of mortality rates and neurological outcomes. According to a systematic analysis by Peterson et al. (2008), the relative risks (RR) of mortality (95\% confidence interval) in comparison with control treatment groups were lower in subgroups of hypothermic procedures which utilized a cooling duration of over 48 hours $(R R=0.51)$, delivered at a temperature range of 33 degrees Celsius and above that was defined as milder than below 33 degrees Celsius $(\mathrm{RR}=0.77)$, and when the patient was rewarmed passively $(\mathrm{RR}=0.49)$. Overall, patients' positive outcomes were more significantly demonstrated over long-term follow-up of at least 1 year. Needless to say, these condition variables and results are an indication or suggestive that a stable maintenance of hypothermia therapy delivered over the mild intensity range, i.e. 33 degrees Celsius and above, produces near to optimal results that are more evidenced over the long term as opposed to substantially strong cooling. Such methodology could help guide treatment standard protocol that can simultaneously increase favorable neurological outcomes and reduce the risk of occurrence of pneumonia and heart rate complications due to milder cooling temperature being introduced as well as a gradual pace of passive rewarming method. This also allows for a broader and more flexible duration window of adjustment and recovery for the brain and peripheral systems while they are still being subjected to potential post-injury effect(s). Building on the concept of stability and mild intensity cooling, a method of less invasive and indirect delivery of hypothermia therapy yet permitting a greater amount of physical surface area of exposure, through placement of patient in low environmental temperature recovery units may be worth considering. No doubt, there is a broad range of interrelated physiological aspects and processes which are subjected to constant modulatory strategies by temperature dynamics, e.g. immune system directed inflammation, increased intracranial pressure, etc., and such associations increase the 
chances of their resultant pathological conditions being potentially treatable and managed by hypothermia treatment [2].

The Eurotherm3235 Trial study conducted by Andrews et al. (2018) [3] to investigate the effectiveness of therapeutic hypothermia in reducing intracranial pressure (ICP) and improving outcomes of patients with severe traumatic brain injury (TBI) reported more negative results for patients in the hypothermia treatment intervention group in terms of mortality rate and functional outcomes. Ruling out raised intracranial pressure as a cause of mortality may offer a more definitive conclusion report to this trial, which has been met with poor unexpected outcomes for patients who received the treatment; however, with critical care needed for a severe grade of traumatic brain injury, the authors should also consider other factors potentiating a broader range of complications for these patients, in addition to raised ICP, that could have exposed both treatment and control groups to higher risks of mortality. In the case of this trial, raised ICP as a sole target in hypothermia treatment has a questionable basis and role in longterm prognosis of TBI. It also fails to guide the establishment of a standardized confident range of temperature target for cooling procedures approved for TBI care and management with a clear understanding of the underlying mechanism through which lowered ICP was achieved. The mild range of 35 to 32 degrees Celsius was beneficial for ICP control but lacks utility for, and/or may even exacerbate, secondary complications during and beyond ICP monitoring. ICP monitoring could be considered as neither beneficial nor harmful in other situational and geographical contexts involving TBI, although it is recommended based on retrospective evidence [4]. Surprising reduction in survival rates have been reported in certain cases, which may partially explain or support the results of this trial. On the other hand, in consideration of the significance of intracranial pressure as an independent variable for recovery from TBI as it has been in this study, there was no mention of the presence or absence of post-traumatic refractory intracranial hypertension in patient subjects' follow-up analyses, which may necessitate more critical intervention strategies and procedures post-treatment [5].

\section{Acknowledgement}

The authors whose names are listed above certify that they have NO affiliations with or involvement in any organization or entity with any financial interest (such as honoraria; educational grants; participation in speakers' bureaus; membership, employment, consultancies, stock ownership, or other equity interest; and expert testimony or patent-licensing arrangements), or non-financial interest (such as personal or professional relationships, affiliations, knowledge or beliefs) in the subject matter or materials discussed in this manuscript.

\section{References}

[1] Peterson K, Carson S, Carney N. Hypothermia treatment for traumatic brain injury: a systematic review and meta-analysis. J Neurotrauma. 2008 Jan;25(1):62-71. [PMID: 18355159]

[2] Tomura S, de Rivero Vaccari JP, Keane RW, Bramlett HM, Dietrich WD. Effects of therapeutic hypothermia on inflammasome signaling after traumatic brain injury. J Cereb Blood Flow Metab. 2012 Oct;32(10):1939-47. [PMID: 22781337]

[3] Andrews PJ, Sinclair HL, Rodríguez A, Harris B, Rhodes J, Watson H, Murray G. Therapeutic hypothermia to reduce intracranial pressure after traumatic brain injury: the Eurotherm3235 RCT. Health Technol Assess. 2018 Aug;22(45):1-134. [PMID: 30168413]

[4] Adams CA, Stein DM, Morrison JJ, Scalea TM. Does intracranial pressure management hurt more than it helps in traumatic brain injury? Trauma Surg Acute Care Open. 2018 Jan 12;3(1):eooo142. [PMID: 29766131]

[5] Bor-Seng-Shu E, Paiva WS, Figueiredo EG, Fujimoto Y, de Andrade AF, Fonoff ET, Teixeira MJ. Posttraumatic refractory intracranial hypertension and brain herniation syndrome: cerebral hemodynamic assessment before decompressive craniectomy. Biomed Res Int. 2013;2013:750809. [PMID: 24377095] 
Citation: Tieng Kong RL. Therapeutic Hypothermia: Its Potential and Questionable Role in Traumatic Brain Injury. Asp Biomed Clin Case Rep. 2019 Oct 30;2(2):84-86.

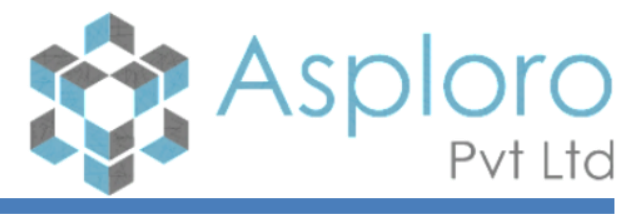

Keywords: Therapeutic Hypothermia; Temperature; Cooling; Intracranial Hypertension; Traumatic Brain Injury 